\title{
Real Hypersurfaces in Complex Hyperbolic Space with Com- muting Ricci Tensor
}

\author{
U-HANG KI \\ The National Academy of Sciences, Seoul 137-044, Korea \\ e-mail : uhangki2005@yahoo.co.kr \\ YOUNG JIN SUH \\ Department of Mathematics, Kyungpook National University, Daegu 702-701, Ko- \\ rea \\ e-mail : yjsuh@knu.ac.kr
}

Abstract. In this paper we consider a real hypersurface $M$ in complex hyperbolic space $H_{n} \mathbb{C}$ satisfying $S \phi A=\phi A S$, where $\phi, A$ and $S$ denote the structure tensor, the shape operator and the Ricci tensor of $M$ respectively. Moreover, we give a characterization of real hypersurfaces of type $\mathrm{A}$ in $H_{n} \mathbb{C}$ by such a commuting Ricci tensor.

\section{Introduction}

A complex $n$-dimensional Kaehler manifold of constant holomorphic sectional curvature $c$ is called a complex space form, which is denoted by $M_{n}(c)$. As is wellknown, a connected complete and simply connected complex space form is complex analytically isometric to a complex projective space $P_{n} \mathbb{C}$, a complex Euclidean space $\mathbb{C}^{n}$ or a complex hyperbolic space $H_{n} \mathbb{C}$ according as $c>0, c=0$ or $c<0$.

Let $M$ be a real hypersurface in $M_{n}(c)$. Then $M$ has an almost contact metric structure $(\phi, \xi, \eta, g)$ induced from the Kaehler structure $J$ and the Kaehlerian metric $G$ of $M_{n}(c)$. The structure vector field $\xi$ is said to be principal if $A \xi=\alpha \xi$ holds on $M$, where $A$ denotes the shape operator of $M$ in $M_{n}(c)$ and $\alpha=\eta(A \xi)$. $\mathrm{A}$ real hypersurface is said to be a Hopf hypersurface if the structure vector field $\xi$ of $M$ is principal. For examples of such kind of Hopf hypersurfaces in $P_{n} \mathbb{C}$ we give some homogeneous real hypersurfaces which are represented as orbits under certain subgroup of the projective unitary group $P U(n+1)([8])$.

Berndt [1] showed that all real hypersurfaces with constant principal curvature of a complex hyperbolic space $H_{n} \mathbb{C}$ are realized as the tubes of constant radius over certain submanifolds when the structure vector field $\xi$ is principal. Nowadays in

Received October 11, 2007.

2000 Mathematics Subject Classification: 53C40, 53C15.

Key words and phrases: real hypersurface, Ricci tensor, Hopf hypersurface.

The second author was supported by grant Proj. No. R17-2008-001-01001 from Korea Science \& Engineering Foundation and by grant Proj. No. KRF-2007-313-C00058 from Korea Research Foundation 
$H_{n} \mathbb{C}$ they said to be of type $\left(\mathrm{A}_{0}\right),\left(\mathrm{A}_{1}\right),\left(\mathrm{A}_{2}\right)$ and $(\mathrm{B})$. He proved the following :

Theorem B ([1]). Let $M$ be a real hypersurface in $H_{n} \mathbb{C}$. Then $M$ has constant principal curvatures and $\xi$ is principal if and only if $M$ is locally congruent to one of the following :

$\left(\mathrm{A}_{0}\right)$ a self-tube, that is, a horosphere,

$\left(\mathrm{A}_{1}\right)$ a geodesic hypersphere or a tube over a hyperplane $H_{n-1} \mathbb{C}$,

$\left(\mathrm{A}_{2}\right)$ a tube over a totally geodesic $H_{k} \mathbb{C}(1 \leq k \leq n-2)$,

(B) a tube over a totally real hyperbolic space $H_{n} \mathbb{R}$.

On the other hand, we remark that every homogeneous real hypersurface in $P_{n} \mathbb{C}$ was proved a Hopf hypersurface (cf. [2], [8]). However, in $H_{n} \mathbb{C}$ there exists some kinds of homogeneous real hypersurfaces, called ruled real hypersurfaces, which are not Hopf hypersurfaces (see [6]).

Let $M$ be a real hypersurface of type $\left(\mathrm{A}_{0}\right),\left(\mathrm{A}_{1}\right)$ or $\left(\mathrm{A}_{2}\right)$ in a complex hyperbolic space $H_{n} \mathbb{C}$. Now, hereafter unless otherwise stated, such hypersurfaces are said to be of type A for our convenience sake. Now we introduce a theorem due to Montiel and Romero [7] as follows:

Theorem MR ([7]). If the shape operator $A$ and the structure operator $\phi$ commute to each other, then a real hypersurface of a complex hyperbolic space $H_{n} \mathbb{C}$ is locally congruent to be of type $\mathrm{A}$.

Now let us denote by $S$ the Ricci tensor of $M$ in a complex space form $M_{n}(c)$. Then in a paper due to Kwon and the second author [3], they considered a real hypersurface $M$ in a complex space form $M_{n}(c)$ with $\mathcal{L}_{\xi} S=\nabla_{\xi} S$, where $\mathcal{L}_{\xi}$ and $\nabla_{\xi}$ respectively denotes the Lie derivative and the covariant derivative along the direction of the structure vector $\xi$ of $M$. Then it was proved that $\mathcal{L}_{\xi} S=\nabla_{\xi} S$ is equivalent to the condition $S \phi A=\phi A S$.

In such a case we say that $M$ has commuting Ricci tensor. That is, the Ricci tensor $S$ of $M$ in $M_{n}(c)$ commutes with the tensor $\phi A$.

Now let us consider a real hypersurface $M$ in $M_{n}(c)$ with $S \phi A-\phi A S=0$. Then we have (see [5])

$$
\|S \phi-\phi S\|^{2}+\frac{3}{2} c\|\phi A \xi\|^{2}=0 .
$$

From this naturally $M$ becomes a Hopf hypersurface if $c>0$. In the case where $c<0$, by using the method of $A^{2} \xi \equiv 0(\bmod \xi, A \xi)$, Kwon and the second author ([3]) proved the following :

Theorem KS ([3]). Let $M$ be a real hypersurface in $H_{n} \mathbb{C}, n \geq 3$, with commuting Ricci tensor. If the structure vector field $\xi$ is principal, then $M$ is locally congruent to of type $\mathrm{A}$.

Then we want to make a generalization of Theorem KS without the assumption that the structure vector field $\xi$ is principal. In this paper we have introduced 
a certain vector $U$ defined by $U=\nabla_{\xi} \xi$ and have applied such a vector to the expression of $A^{2} \xi \equiv 0(\bmod \xi, A \xi)$, and finally proved that the structure vector $\xi$ is principal. Namely, we prove the following

Theorem. Let $M$ be a real hypersurface in a complex hyperbolic space $H_{n} \mathbb{C}, n \geq 3$, with commuting Ricci tensor. Then $M$ becomes a Hopf hypersurface. Further, $M$ is locally congruent to one of the following spaces :

$\left(\mathrm{A}_{0}\right)$ a self-tube, that is, a horosphere,

$\left(\mathrm{A}_{1}\right)$ a geodesic hypersphere or a tube over a hyperplane $H_{n-1} \mathbb{C}$,

$\left(\mathrm{A}_{2}\right)$ a tube over a totally geodesic $H_{k} \mathbb{C}(1 \leq k \leq n-2)$.

\section{Preliminaries}

Let $M$ be a real hypersurface immersed in a complex space form $\left(M_{n}(c), G\right)$ with almost complex structure $J$ of constant holomorphic sectional curvature $c$, and let $C$ be a unit normal vector field on $M$. The Riemannian connection $\tilde{\nabla}$ in $M_{n}(c)$ and $\nabla$ in $M$ are related by the following formulas for any vector fields $X$ and $Y$ on $M$ :

$$
\begin{gathered}
\tilde{\nabla}_{Y} X=\nabla_{Y} X+g(A Y, X) C, \\
\tilde{\nabla}_{X} C=-A X,
\end{gathered}
$$

where $g$ denotes the Riemannian metric on $M$ induced from that $G$ of $M_{n}(c)$ and $A$ is the shape operator of $M$ in $M_{n}(c)$. A characteristic vector $X$ of the shape operator of $A$ is called a principal curvature vector. Also an eigenvalue $\lambda$ of $A$ is called a principal curvature. It is known that $M$ has an almost contact metric structure induced from the almost complex structure $J$ on $M_{n}(c)$, that is, we define a tensor field $\phi$ of type $(1,1)$, a vector field $\xi$, a 1-form $\eta$ on $M$ by $g(\phi X, Y)=G(J X, Y)$ and $g(\xi, X)=\eta(X)=G(J X, C)$. Then we have

$$
\phi^{2} X=-X+\eta(X) \xi, \quad g(\xi, \xi)=1, \quad \phi \xi=0 .
$$

From (1.1) we see that

$$
\begin{gathered}
\left(\nabla_{X} \phi\right) Y=\eta(Y) A X-g(A X, Y) \xi, \\
\nabla_{X} \xi=\phi A X .
\end{gathered}
$$

Since the ambient space is of constant holomorphic sectional curvature $c$, equations of the Gauss and Codazzi are respectively given by

$$
\begin{gathered}
R(X, Y) Z=\frac{c}{4}\{g(Y, Z) X-g(X, Z) Y+g(\phi Y, Z) \phi X-g(\phi X, Z) \phi Y \\
-2 g(\phi X, Y) \phi Z\}+g(A Y, Z) A X-g(A X, Z) A Y \\
\left(\nabla_{X} A\right) Y-\left(\nabla_{Y} A\right) X=\frac{c}{4}\{\eta(X) \phi Y-\eta(Y) \phi X-2 g(\phi X, Y) \xi\}
\end{gathered}
$$


for any vector fields $X, Y$ and $Z$ on $M$, where $R$ denotes the Riemannian curvature tensor of $M$. We shall denote the Ricci tensor of type $(1,1)$ by $S$. Then it follows from (1.6) that

$$
S X=\frac{c}{4}\{(2 n+1) X-3 \eta(X) \xi\}+h A X-A^{2} X,
$$

where $h=\operatorname{trace} A$.

To write our formulas in convention forms, we denote $\alpha=\eta(A \xi), \beta=\eta\left(A^{2} \xi\right)$, $\gamma=\eta\left(A^{3} \xi\right), \delta=\eta\left(A^{4} \xi\right), \mu^{2}=\beta-\alpha^{2}$ and $\nabla f$ by the gradient vector field of a function $f$ on $M$. In the following, we use the same terminology and notation as above unless otherwise stated.

If we put $U=\nabla_{\xi} \xi$, then $U$ is orthogonal to the structure vector field $\xi$. Then using (1.3) and (1.5), we see that

$$
\phi U=-A \xi+\alpha \xi,
$$

which shows that $g(U, U)=\beta-\alpha^{2}$. By the definition of $U,(1.3)$ and (1.5) it is verify that

$$
g\left(\nabla_{X} \xi, U\right)=g\left(A^{2} \xi, X\right)-\alpha g(A \xi, X) .
$$
find

Now, differentiating (1.9) covariantly along $M$ and using (1.4) and (1.5), we

$$
\begin{aligned}
& \eta(X) g(A U+\nabla \alpha, Y)+g\left(\phi X, \nabla_{Y} U\right) \\
& \quad=g\left(\left(\nabla_{Y} A\right) X, \xi\right)-g(A \phi A X, Y)+\alpha g(A \phi X, Y),
\end{aligned}
$$

which enables us to obtain

$$
\left(\nabla_{\xi} A\right) \xi=2 A U+\nabla \alpha
$$

because of (1.7). From (1.11) we also have

$$
\nabla_{\xi} U=3 \phi A U+\alpha A \xi-\beta \xi+\phi \nabla \alpha,
$$

where we have used (1.3), (1.5) and (1.10).

We put

$$
A \xi=\alpha \xi+\mu W,
$$

where $W$ is a unit vector field orthogonal to $\xi$. Then from (1.9) it is seen that $U=\mu \phi W$ and hence $g(U, U)=\mu^{2}$, and $W$ is also orthogonal to $U$. Thus, we see, making use of (1.5), that

$$
\mu g\left(\nabla_{X} W, \xi\right)=g(A U, X) .
$$




\section{Real hypersurfaces satisfying $A^{2} \xi \equiv 0(\bmod \xi, A \xi)$}

Let $M$ be a real hypersurface of a complex space form $M_{n}(c), c \neq 0$. If it satisfies $A^{2} \xi \equiv 0(\bmod \xi, A \xi)$. So we can put

$$
A^{2} \xi=\rho A \xi+(\beta-\rho \alpha) \xi
$$

for a certain scalar $\rho$.

Hereafter, unless otherwise stated, let us assume that $\mu \neq 0$ on $M$, that is, $\xi$ is not a principal curvature vector field and we put $\Omega=\{p \in M \mid \mu(p) \neq 0\}$. Then $\Omega$ is an open subset of $M$, and from now on we discuss our arguments on $\Omega$.

From (1.14) and (2.1), we see that

$$
A W=\mu \xi+(\rho-\alpha) W
$$

and hence

$$
A^{2} W=\rho A W+(\beta-\rho \alpha) W
$$

because $\mu \neq 0$.

Now, differentiating (2.2) covariantly along $\Omega$, we find

$$
\left(\nabla_{X} A\right) W+A \nabla_{X} W=(X \mu) \xi+\mu \nabla_{X} \xi+X(\rho-\alpha) W+(\rho-\alpha) \nabla_{X} W .
$$

By taking an inner product with $W$ in the last equation, we obtain

$$
g\left(\left(\nabla_{X} A\right) W, W\right)=-2 g(A X, U)+X \rho-X \alpha
$$

since $W$ is a unit vector field orthogonal to $\xi$. We also have by applying $\xi$ to (2.4)

$$
\mu g\left(\left(\nabla_{X} A\right) W, \xi\right)=(\rho-2 \alpha) g(A U, X)+\mu(X \mu),
$$

where we have used (1.15), which together with the Codazzi equation (1.7) gives

$$
\begin{aligned}
& \mu\left(\nabla_{W} A\right) \xi=(\rho-2 \alpha) A U-\frac{c}{2} U+\mu \nabla \mu, \\
& \mu\left(\nabla_{\xi} A\right) W=(\rho-2 \alpha) A U-\frac{c}{4} U+\mu \nabla \mu .
\end{aligned}
$$

Replacing $X$ by $\xi$ in (2.4) and taking account of (2.8), we find

$$
\begin{gathered}
(\rho-2 \alpha) A U-\frac{c}{4} U+\mu \nabla \mu+\mu\left\{A \nabla_{\xi} W-(\rho-\alpha) \nabla_{\xi} W\right\} \\
=\mu(\xi \mu) \xi+\mu^{2} U+\mu(\xi \rho-\xi \alpha) W .
\end{gathered}
$$

On the other hand, from $\phi U=-\mu W$ we have

$$
g(A U, X) \xi-\phi \nabla_{X} U=(X \mu) W+\mu \nabla_{X} W .
$$


Replacing $X$ by $\xi$ in this and using (1.9) and (1.13), we get

$$
\mu \nabla_{\xi} W=3 A U-\alpha U+\nabla \alpha-(\xi \alpha) \xi-(\xi \mu) W,
$$

which implies

$$
W \alpha=\xi \mu .
$$

From the last three equations, it follows that

$$
\begin{aligned}
& 3 A^{2} U-2 \rho A U+A \nabla \alpha+\frac{1}{2} \nabla \beta-\rho \nabla \alpha+\left(\alpha \rho-\beta-\frac{c}{4}\right) U \\
& =2 \mu(W \alpha) \xi+\mu(\xi \rho) W-(\rho-2 \alpha)(\xi \alpha) \xi,
\end{aligned}
$$

which enables us to obtain

$$
\xi \beta=2 \alpha(\xi \alpha)+2 \mu(W \alpha) .
$$

Differentiating (2.1) covariantly and making use of (1.5), we get

$$
\begin{aligned}
& \left(\nabla_{X} A\right) A \xi+A\left(\nabla_{X} A\right) \xi+A^{2} \phi A X-\rho A \phi A X \\
& =(X \rho) A \xi+\rho\left(\nabla_{X} A\right) \xi+X(\beta-\rho \alpha) \xi+(\beta-\rho \alpha) \phi A X,
\end{aligned}
$$

which together with (1.7) implies that

$$
\begin{aligned}
& \frac{c}{4}\{u(Y) \eta(X)-u(X) \eta(Y)\}+\frac{c}{2}(\rho-\alpha) g(\phi Y, X)-g\left(A^{2} \phi A X, Y\right) \\
& +g\left(A^{2} \phi A Y, X\right)+2 \rho g(\phi A X, A Y)-(\beta-\rho \alpha)\{g(\phi A Y, X)-g(\phi A X, Y)\} \\
& =g\left(A Y,\left(\nabla_{X} A\right) \xi\right)-g\left(A X,\left(\nabla_{Y} A\right) \xi\right)+(Y \rho) g(A \xi, X)-(X \rho) g(A \xi, Y) \\
& \quad+Y(\beta-\rho \alpha) \eta(X)-X(\beta-\rho \alpha) \eta(Y)
\end{aligned}
$$

where we have defined a 1 -form $u$ by $u(X)=g(U, X)$ for any vector field $X$. If we replace $X$ by $\mu W$ to the both sides of (2.15) and take account of (1.12), (2.2), (2.3), (2.6) and (2.7), we obtain

$$
\begin{gathered}
(3 \alpha-2 \rho) A^{2} U+2\left(\rho^{2}+\beta-2 \rho \alpha+\frac{c}{4}\right) A U+(\rho-\alpha)\left(\beta-\rho \alpha-\frac{c}{2}\right) U \\
=\mu A \nabla \mu+(\alpha \rho-\beta) \nabla \alpha-\frac{1}{2}(\rho-\alpha) \nabla \beta+\mu^{2} \nabla \rho \\
-\mu(W \rho) A \xi-\mu W(\beta-\rho \alpha) \xi .
\end{gathered}
$$

Using (1.14), we can write the equation (2.14) as

$$
\begin{aligned}
& A\left(\nabla_{X} A\right) \xi+(\alpha-\rho)\left(\nabla_{X} A\right) \xi+\mu\left(\nabla_{X} A\right) W \\
& =(X \rho) A \xi+X(\beta-\rho \alpha) \xi+(\beta-\rho \alpha) \phi A X+\rho A \phi A X-A^{2} \phi A X .
\end{aligned}
$$


Thus, from this, by replacing $X$ by $\alpha \xi+\mu W$ and making use of (1.5), (1.12), (1.14) and $(2.5) \sim(2.7)$, we find

$$
\begin{aligned}
& 2 \rho A^{2} U+2\left(\alpha \rho-\beta-\rho^{2}-\frac{c}{4}\right) A U+\left(\rho^{2} \alpha-\rho \beta+\frac{c}{2} \rho-\frac{3}{4} c \alpha\right) U \\
& =g(A \xi, \nabla \rho) A \xi-\frac{1}{2} A \nabla \beta+\frac{1}{2}(\rho-2 \alpha) \nabla \beta+\beta \nabla \alpha \\
& -\mu^{2} \nabla \rho+g(A \xi, \nabla(\beta-\rho \alpha)) \xi .
\end{aligned}
$$

\section{Real hypersurfaces in $H_{n} \mathbb{C}$ with commuting Ricci tensor}

Let us consider a real hypersurface $M$ in complex hyperbolic space $H_{n} \mathbb{C}$ with negative constant holomorphic sectional curvature $c<0$. If $M$ satisfies $S \phi A-$ $\phi A S=0$, we say that $M$ has commuting Ricci tensor. In this section we consider a real hypersurface $M$ in $H_{n} \mathbb{C}$ with commuting Ricci tensor. Then by (1.8) we have

$$
h\left(A \phi A-\phi A^{2}\right)+\phi A^{3}-A^{2} \phi A+\frac{3}{4} c \eta \otimes U=0,
$$

where we have used (1.5). Taking the transpose of this, we find

$$
h\left(A \phi A-A^{2} \phi\right)+A^{3} \phi-A \phi A^{2}+\frac{3}{4} c U \otimes \xi=0 .
$$

Transforming (3.1) by $A$ to the left, and (3.2) to the right respectively, and combining to these two equations, we obtain

$$
\eta \otimes A U+\xi \otimes \eta(A \phi A)=0
$$

which implies

$$
A U=0
$$

If we take an inner product (3.2) with $\xi$ and make use of (3.3), then we have

$$
A \phi A^{2} \xi=0 \text {. }
$$

Taking an inner product (3.1) with $\xi$ and using (3.3) and the last equation, we also find

$$
\phi\left(A^{3} \xi-h A^{2} \xi\right)+\frac{3}{4} c U=0 .
$$

If we apply this by $\phi$ and take account of (1.9), then we get

$$
A^{3} \xi-h A^{2} \xi=\left(\gamma-\beta h+\frac{3}{4} c \alpha\right) \xi-\frac{3}{4} c A \xi,
$$

which tells us that

$$
A^{4} \xi-h A^{3} \xi=\left(\gamma-\beta h+\frac{3}{4} c \alpha\right) A \xi-\frac{3}{4} c A^{2} \xi .
$$


Next, applying (3.1) by $A \xi$ and making use of (3.3) and (3.4), we have

$$
\phi\left(A^{4} \xi-h A^{3} \xi\right)=\frac{3}{4} c \alpha U,
$$

which implies that

$$
A^{4} \xi-h A^{3} \xi=-\frac{3}{4} c \alpha(A \xi-\alpha \xi)+(\delta-h \gamma) \xi .
$$

This, together with (3.5) implies that

$$
\frac{3}{4} c A^{2} \xi=\left(\gamma-\beta h+\frac{3}{2} c \alpha\right) A \xi+\left(h \gamma-\delta-\frac{3}{4} c \alpha^{2}\right) \xi .
$$

Thus, it follows that

$$
\frac{3}{4} c\left(\beta-\alpha^{2}\right)=\alpha(\gamma-\beta h)+h \gamma-\delta .
$$

Therefore (3.6) is reformed as

$$
A^{2} \xi=\rho A \xi+(\beta-\rho \alpha) \xi,
$$

where the function $\rho$ is defined in such a way that

$$
\frac{3}{4} c \rho=\gamma-\beta h+\frac{3}{2} c \alpha .
$$

Accordingly the formulas stated in Section 2 are established.

Now, we are going to prove our Main Theorem.

Transforming (2.12) by $U$ and using (3.3), we find

$$
\frac{1}{2} U \beta-\rho(U \alpha)=\left(\beta-\rho \alpha+\frac{c}{4}\right) \mu^{2} .
$$

Similarly, from (2.16) and (2.17) we have respectively

$$
\begin{gathered}
(\alpha \rho-\beta) U \alpha-\frac{1}{2}(\rho-\alpha) U \beta+\left(\beta-\alpha^{2}\right) U \rho=(\rho-\alpha)\left(\beta-\rho \alpha-\frac{c}{2}\right) \mu^{2}, \\
\frac{1}{2}(\rho-2 \alpha) U \beta+\beta(U \alpha)-\left(\beta-\alpha^{2}\right) U \rho=\left(\rho^{2} \alpha-\rho \beta+\frac{c}{2} \rho-\frac{3}{4} c \alpha\right) \mu^{2} .
\end{gathered}
$$

Differentiating (3.3) covariantly along $\Omega$, we find

$$
\left(\nabla_{X} A\right) U+A \nabla_{X} U=0 .
$$

If we put $X=\xi$ in this and take account of (1.13) and (3.3), we obtain

$$
\left(\nabla_{\xi} A\right) U+\alpha A^{2} \xi-\beta A \xi+\alpha A \phi \nabla \alpha=0,
$$


which shows that

$$
\phi\left(\nabla_{\xi} A\right) U=(\beta-\rho \alpha) U-\alpha \phi A \phi \nabla \alpha,
$$

where we have used (3.8). From this and (1.7), it follows that

$$
\phi\left(\nabla_{U} A\right) \xi=\left(\beta-\rho \alpha+\frac{c}{4}\right) U-\alpha \phi A \phi \nabla \alpha .
$$

On the other hand, from $\nabla_{X} \xi=\phi A X$ and $U=\nabla_{\xi} \xi$, we see that

$$
\nabla_{X} U=\phi\left(\nabla_{X} A\right) \xi+\alpha A X-g\left(A^{2} X, \xi\right) \xi+\phi A \phi A X
$$

by virtue of (1.4). Replacing $X$ by $U$ in this and making use of (3.3), we obtain $\nabla_{U} U=\phi\left(\nabla_{U} A\right) \xi$, which together with (3.13) implies that

$$
\nabla_{U} U=\left(\beta-\rho \alpha+\frac{c}{4}\right) U-\alpha \phi A \phi \nabla \alpha .
$$

If we take an inner product with $U$ to the last equation and use (1.9), (3.3) and $\mu^{2}=\beta-\alpha^{2}$, then we get

$$
\frac{1}{2} U \beta-\alpha(U \alpha)=\left(\beta-\rho \alpha+\frac{c}{4}\right) \mu^{2} .
$$

This, together with (3.10), implies that

$$
(\rho-\alpha) U \alpha=-2\left(\beta-\rho \alpha+\frac{c}{4}\right) \mu^{2} .
$$

Combining (3.12) to (3.14), we find

$$
U \rho=U \alpha-\frac{c}{4}(\rho-\alpha)
$$

Substituting (3.14), (3.15) and (3.16) into (3.12), we obtain $\rho-\alpha=0$ and hence $\beta-\alpha^{2}+\frac{c}{4}=0$ by virtue of (3.15). Thus, (3.8) becomes $A^{2} \xi=\alpha A \xi-\frac{c}{4} \xi$, which tells us that $\gamma=\alpha^{3}-\frac{c}{2} \alpha$. Then it follows

$$
\delta=\alpha^{4}-\frac{3}{4} c \alpha^{2}+\left(\frac{c}{4}\right)^{2} .
$$

Using above facts, (3.7) turns out to be

$$
\alpha h=\alpha^{2}+\frac{c}{2} .
$$

Since $\rho=\alpha,(3.9)$ becomes $\gamma-\beta h=-\frac{3}{4} c \alpha$, which implies that $\alpha^{3}-h\left(\alpha^{2}-\frac{c}{4}\right)=$ $-\frac{c}{4} \alpha$. This, together with (3.17), yields $c=0$, a contradiction. Hence $\Omega=\emptyset$. Thus, the subset $\Omega$ (of $M$ ) on which $A \xi-\eta(A \xi) \xi \neq 0$ is an empty set, namely in $H_{n} \mathbb{C}$ every real hypersurface satisfying $S \phi A=\phi A S$ is a Hopf hypersurface. Then, by Theorem KS we complete the proof of our Main Theorem. 


\section{References}

[1] J. Berndt, Real hypersurfaces with constant principal curvatures in a complex hyperbolic space, J. Reine Agnew. Math. 395(1989), 132-141.

[2] T. E. Cecil and P.J. Ryan, Focal sets and real hypersurfaces in complex projective space, Trans. Amer. Math. Soc. 269(1982), 481-499.

[3] J.-H. Kwon and Y.J. Suh, A new characterization of homogeneous real hypersurfaces in complex space forms, Nihonkai Math. J. 9(1998), 77-90.

[4] M. Kimura, Real hypersurfaces and complex submanifolds in complex projective space, Trans. Amer. Math. Soc. 296(1986), 137-149.

[5] M. Kimura and S. Maeda, Lie derivatives on real hypersurfaces in a complex projective space, Czechoslovak Math. J. 45(1995), 135-148.

[6] M. Lohnherr and H. Reckziegel, On ruled real hypersurfaces in complex space forms, Geom. Dedicata 74(1999), 267-286.

[7] S. Montiel and A. Romero, On some real hypersurfaces of a complex hyperbolic space, Geom. Dedicata 20(1986), 245-261.

[8] R. Takagi, On homogeneous real hypersurfaces in a complex projective space, Osaka J. Math. 10(1973), 495-506. 